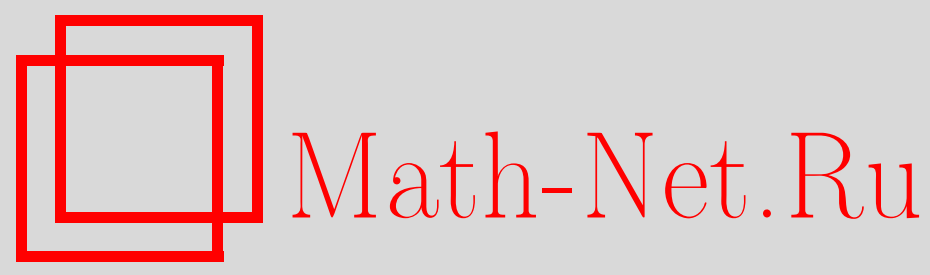

Ю. Л. Павлов, О предельных распределениях степеней вершин в условных Интернет-графах, Дискрет. матем., 2009, том 21, выпуск 3, 14-23

DOI: https://doi.org/10.4213/dm1057

Использование Общероссийского математического портала Math-Net.Ru подразумевает, что вы прочитали и согласны с пользовательским соглашением http://www . mathnet.ru/rus/agreement

Параметры загрузки:

IP: 54.162 .127 .20

26 апреля 2023 г., 02:12:44 


\title{
О предельных распределениях степеней вершин в условных Интернет-графах
}

\author{
() 2009 г. Ю. Л. Павлов
}

\begin{abstract}
Рассматриваются случайные графы, моделирующие структуру больших сетей передачи данных, включая Интернет. Изучается подмножество таких графов, состоящих из $N$ вершин при условии, что число ребер равно $n$. Получены предельные распределения максимальной степени вершины и числа вершин заданной степени при $N, n \rightarrow \infty$ так, что $n / N \rightarrow \lambda$, где $\lambda-$ положительная постоянная.

Работа выполнена при поддержке Российского фонда фундаментальных исследований, проект 05-01-00007а, и программы Президента Российской Федерации поддержки ведущих научных школ, грант НШ 4129.2006.1.
\end{abstract}

В статьях [1, 2] рассматривался известный (см., например, [3]) вид случайного графа, предназначенный для моделирования сложных сетей телекоммуникаций, в частности, сети Интернет. В связи с этим такие графы иногда называют Интернет-графами. Предполагается, что граф состоит из $N$ основных вершин, занумерованных числами от 1 до $N$, и содержит еще одну вспомогательную вершину, то есть общее число вершин графа равно $N+1$. Степени основных вершин задаются независимыми случайными величинами $\eta_{1}, \ldots, \eta_{N}$, имеющими общее распределение, являющееся дискретным аналогом распределения Парето с положительным показателем $\tau$ :

$$
\mathbf{P}\left\{\eta_{1} \geqslant k\right\}=k^{-\tau}, \quad k=1,2, \ldots
$$

При описании структуры Интернет-графа в [3] введено понятие полуребра вершины, то есть ребра, инцидентного данной вершине, для которого смежная вершина еще не определена. Все полуребра графа считаются различными и при образовании ребер соединяются равновероятно. Для того, чтобы сумма степеней вершин была четной, вспомогательная вершина имеет степень 0 или 1 в зависимости от того, является ли сумма степеней основных вершин четной или нет.

Во многих работах (см., например, $[3,4,5])$ изучалось предельное поведение различных характеристик Интернет-графов при $N \rightarrow \infty, 1<\tau<2$. Выбор интервала $(1,2)$ для параметра $\tau$ объясняется тем, что наблюдения за реальными сетями (см. $[4,5])$ показали, что такие значения $\tau$ являются типичными. В то же время отмечается, что при $\tau=1,2$ происходят существенные изменения свойств случайных графов. Поэтому представляет интерес изучение Интернет-графов и при значениях параметра $\tau$ вне интервала $(1,2)$.

В $[1,2]$ впервые предложено использовать обобщенную схему размещения частиц по ячейкам с целью исследования асимптотического поведения Интернет-графов при всех 
значениях $\tau$. Обобщенная схема была введена и изучена В. Ф. Колчиным (см., например, [6]). Из (1) следует, что

$$
p_{k}=\mathbf{P}\left\{\eta_{1}=k\right\}=k^{-\tau}-(k+1)^{-\tau}, \quad k=1,2, \ldots
$$

Пусть

$$
v_{N}=\eta_{1}+\ldots+\eta_{N}
$$

Введем вспомогательные независимые одинаково распределенные случайные величины $\xi_{1}, \ldots, \xi_{N}$, для которых

$$
p_{k}(\lambda)=\mathbf{P}\left\{\xi_{1}=k\right\}=\lambda^{k} p_{k}(1-(1-\lambda) \Phi(\lambda, \tau, 1))^{-1},
$$

где $k=1,2, \ldots, 0<\lambda<1$,

$$
\Phi(z, s, a)=\sum_{j=0}^{\infty} \frac{z^{j}}{(j+a)^{s}}=\frac{1}{\Gamma(s)} \int_{0}^{\infty} \frac{t^{s-1} e^{-a t}}{1-z e^{-t}} d t,
$$

$\Gamma(s)$ - значение гамма-функции в точке $s$.

Мы будем рассматривать подмножество Интернет-графов, для которых сумма степеней $v_{N}$ известна и равна $n$. Из (2)-(4) нетрудно получить, что для такого множества графов при всех $\lambda, 0<\lambda \leqslant 1$, справедливо равенство

$$
\mathbf{P}\left\{\eta_{1}=k_{1}, \ldots, \eta_{N}=k_{N}\right\}=\mathbf{P}\left\{\xi_{1}=k_{1}, \ldots, \xi_{N}=k_{N} \mid \xi_{1}+\ldots+\xi_{N}=n\right\} .
$$

Это равенство означает, что условия обобщенной схемы размещения [6] выполнены и мы можем ее использовать для исследования условных Интернет-графов при условии $v_{N}=n$.

Обозначим $\eta_{(N)}$ максимальную степень вершины и $\mu_{r}$ число вершин степени $r$. В [1] получены предельные распределения этих случайных величин при $N, n \rightarrow \infty$ так, что $1<C_{1} \leqslant n / N \leqslant C_{2}<\zeta(\tau)$, где $\zeta(\tau)$ - значение дзета-функции Римана в точке $\tau$. В [2] рассматривались также случаи $n / N \downarrow 1$ и $n / N \uparrow \zeta(\tau)$. В этих работах параметр $\lambda$ распределения (3) выбирался как решение уравнения

$$
m(\lambda)=n / N,
$$

где

$$
m(\lambda)=\mathbf{E} \xi_{1}=\frac{\lambda \Phi(\lambda, \tau, 1)-(1-\lambda) \Phi(\lambda, \tau-1,1)}{1-(1-\lambda) \Phi(\lambda, \tau, 1)} .
$$

Используя (2)-(7), нетрудно показать, что если $n / N \leqslant C_{2}<\zeta(\tau)$, то справедливо неравенство $\lambda<1$, при этом из условия $n / N \uparrow \zeta(\tau)$ следует, что $\lambda \uparrow 1$. Для того, чтобы описать предельное поведение $\eta_{(N)}$ и $\mu_{r}$ в случае $n / N \uparrow \zeta(\tau)$, в [2] значения параметров $\tau, N, n$ названы допустимыми для $\lambda$ (являющегося решением уравнения (6)), если выполнено одно из условий:

1. $\tau>3$;

2. $\tau=3,(\ln (1-\lambda)) / \sqrt{N} \rightarrow 0$;

3. $2<\tau<3, \sqrt{N}(1-\lambda)^{3-\tau} \rightarrow \infty$; 
4. $\tau=2, N(1-\lambda)^{2}|\ln (1-\lambda)|^{3} \rightarrow \infty$;

5. $0<\tau<2, N(1-\lambda)^{\tau} \rightarrow \infty$.

Заметим, что условия 4 и 5 являются менее жесткими, чем в [2], но, как нетрудно проверить, доказательства соответствующих результатов в [2] остаются в силе и при таких условиях. Ниже эти результаты приведены в виде теорем 1-3.

Теорема 1. Пусть $N, n \rightarrow \infty$ так, что $n / N \uparrow \zeta(\tau)$ и значения параметров $\tau, N, n$ допустимы для $\lambda$. Пусть $r=r(N, n)$ выбрано так, что

$$
\frac{\tau N \lambda^{r}}{r^{\tau+1}(1-\lambda)} \rightarrow \gamma
$$

где $\gamma$ - некоторая положительная постоянная. Тогда

$$
\mathbf{P}\left\{\eta_{(N)} \leqslant r\right\} \rightarrow e^{-\gamma}
$$

Теорема 2. Пусть $N, n \rightarrow \infty$ так, что $n / N \uparrow \zeta(\tau)$ и значения параметров $\tau, N$, $n$ допустимь для $\lambda$. Тогда для цеельх неотрищательных $k$ равномерно относительно $u_{r}=\left(k-N p_{r}(\lambda)\right) /\left(\sigma_{r r}(\lambda) \sqrt{N}\right)$ в любом фиксированном конечном интервале

$$
\mathbf{P}\left\{\mu_{r}=k\right\}=\left(\sigma_{r r}(\lambda) \sqrt{2 \pi N}\right)^{-1} e^{-u_{r}^{2} / 2}(1+o(1)),
$$

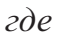

$$
\begin{aligned}
\sigma_{r r}^{2}(\lambda) & =p_{r}(\lambda)\left(1-p_{r}(\lambda)-\frac{(m(\lambda)-r)^{2}}{\sigma^{2}(\lambda)} p_{r}(\lambda)\right), \\
\sigma^{2}(\lambda) & =\mathbf{D} \xi_{1}=\frac{2 \Phi(\lambda, \tau-1,1)-(1-\lambda) \Phi(\lambda, \tau-2,1)-\Phi(\lambda, \tau, 1)}{1-(1-\lambda) \Phi(\lambda, \tau, 1)}-m^{2}(\lambda) .
\end{aligned}
$$

Теорема 3. Пусть выполнены условия теоремы $2 u$, кроме того, $r \rightarrow \infty$. Тогда для ияельх неотрицательных $k$

$$
\mathbf{P}\left\{\mu_{r}=k\right\}=\frac{1}{k !}\left(N p_{r}(\lambda)\right)^{k} \exp \left\{-N p_{r}(\lambda)\right\}(1+o(1))
$$

равномерно относительно $\left(k-N p_{r}(\lambda)\right) / \sqrt{N p_{r}(\lambda)}$ в любом фиксированном конечном интервале.

Используя (2), (3), (4), (7), (9), нетрудно получить, что

$$
\begin{aligned}
m & =m(1)=\mathbf{E} \eta_{1}=\zeta(\tau), \\
\sigma^{2} & =\sigma^{2}(1)=\mathbf{D} \eta_{1}=2 \zeta(\tau-1)-\zeta(\tau)-\zeta^{2}(\tau) .
\end{aligned}
$$

Из (2), (10) находим, что при $\tau>2$ случайные величины $\eta_{1}, \ldots, \eta_{N}$ имеют конечное математическое ожидание и дисперсию. Хорошо известно (см., например, [7]), что в этом случае для суммы $v_{N}$ справедлива локальная предельная теорема, следовательно,

$$
\sup _{k}\left|\sigma \sqrt{2 \pi N} \mathbf{P}\left\{v_{N}=k\right\}-\exp \left\{-\frac{(k-N \zeta(\tau))^{2}}{2 \sigma^{2} N}\right\}\right| \rightarrow 0 .
$$


В случае $\tau \leqslant 2$ из (10) следует, что $\eta_{1}$ не имеет дисперсии. При $\tau=2$ нетрудно получить, используя теоремы 2.2.1, 2.6.2 и 4.2.1 книги [7], что

$$
\sup _{k}\left|\sqrt{2 \pi N \ln N} \mathbf{P}\left\{v_{N}=k\right\}-\exp \left\{-\frac{(k-N \zeta(2))^{2}}{2 N \ln N}\right\}\right| \rightarrow 0 .
$$

В статье [8] доказано, что в случае $1<\tau<2$ справедливо соотношение

$$
\sup _{k}\left|N^{1 / \tau} \mathbf{P}\left\{v_{N}=k\right\}-g\left(\frac{k-N \zeta(\tau)}{N^{1 / \tau}}\right)\right| \rightarrow 0,
$$

где $g(x)$ - плотность устойчивого распределения с показателем $\tau$ и характеристической функцией

$$
f(t)=\exp \left\{-|t|^{\tau} \frac{\Gamma(2-\tau)}{\tau-1}\left(\cos \frac{\pi \tau}{2}\right)\left(1+i \frac{t}{|t|} \operatorname{tg} \frac{\pi \tau}{2}\right)\right\} .
$$

Из (11)-(13) видно, что при исследовании предельного поведения Интернет-графов без ограничений на сумму $v_{N}$ в случае $\tau>1$ наибольший интерес представляет центральная зона изменения параметров, в которой $v_{N} / N \rightarrow \zeta(\tau)$. Однако в статьях $[1,2]$ главным образом рассматривался случай $n / N \leqslant C_{2}<\zeta(\tau)$ и лишь частично охвачена левая часть центральной зоны (см. теоремы 1-3), ограниченная допустимыми для $\lambda$ значениями $\tau, N$, $n$. В настоящей работе исследуется предельное поведение $\eta_{(N)}$ и $\mu_{r}$ в случае $n / N \rightarrow \zeta(\tau)$ и фиксированных $r$. Далее мы будем предполагать, что параметр $\lambda$ распределения (3) равен единице. Нетрудно видеть, что в этом случае распределения случайных величин $\xi_{1}$ и $\eta_{1}$ совпадают (ср. (2)-(4)). Справедливы следующие результаты.

Теорема 4. Пусть $N \rightarrow \infty, n / N \rightarrow \zeta(\tau), r=(N / \gamma)^{1 / \tau}(1+o(1))$, где $\gamma-$ некоторая положительная постоянная. Пусть также выполнено одно из следующих условий:

1. $\tau>2, n-\zeta(\tau) N=O(\sqrt{N})$;

2. $\tau=2, n-\zeta(2) N=O(\sqrt{N \ln N})$;

3. $1<\tau<2, n-\zeta(\tau) N=O\left(N^{1 / \tau}\right)$.

Тогда

$$
\mathbf{P}\left\{\eta_{(N)} \leqslant r\right\} \rightarrow e^{-\gamma}
$$

Теорема 5. Пусть $N \rightarrow \infty, n-\zeta(\tau) N=o(\sqrt{N}), \tau>2$. Тогда для ичельхх неотрицุательных $k$ равномерно относительно $u_{r}=\left(k-N p_{r}\right) /\left(\sigma_{r r} \sqrt{N}\right)$ в любом фиксированном конечном интервале

$$
\mathbf{P}\left\{\mu_{r}=k\right\}=\left(\sigma_{r r} \sqrt{2 \pi N}\right)^{-1} e^{-u_{r}^{2} / 2}(1+o(1)),
$$

2de

$$
\sigma_{r r}^{2}=p_{r}\left(1-p_{r}-\frac{(m-r)^{2}}{\sigma^{2}} p_{r}\right)
$$

Теорема 6. Пусть $N \rightarrow \infty$ и выполнено одно из следующих условий:

1. $\tau=2, n-\zeta(2) N=o(\sqrt{N \ln N})$; 
2. $1<\tau<2, n-\zeta(\tau) N=o\left(N^{1 / \tau}\right)$.

Тогда для иельхх неотрииательных $k$

$$
\mathbf{P}\left\{\mu_{r}=k\right\}=\frac{1+o(1)}{\sqrt{2 \pi N p_{r}\left(1-p_{r}\right)}} \exp \left\{-\frac{\left(k-N p_{r}\right)^{2}}{2 N p_{r}\left(1-p_{r}\right)}\right\} .
$$

равномерно относительно $\left(k-N p_{r}\right) / \sqrt{N p_{r}\left(1-p_{r}\right)}$ в любом фиксированном конечном интервале.

Замечание 1. Пусть $n / N \uparrow \zeta(\tau), \tau>1$ и условия теорем 4-6 не выполнены. Тогда, используя (6), (7) и известные (см. $[9,10])$ асимптотические свойства функции (4), нетрудно получить, что значения параметров $\tau, N, n$ являются допустимыми для $\lambda$ и, следовательно, для описания предельного поведения $\eta_{N}$ и $\mu_{r}$ можно использовать теоремы 1-3.

Ниже приводятся вспомогательные утверждения (леммы 1-6), с помощью которых далее будут доказаны теоремы 4-6.

Введем наборы независимых случайных величин $\xi_{1}^{(r)}, \ldots, \xi_{N}^{(r)}$ и $\tilde{\xi}_{1}^{(r)}, \ldots, \tilde{\xi}_{N}^{(r)}$ таких, что

$$
\begin{aligned}
& \mathbf{P}\left\{\xi_{1}^{(r)}=k\right\}=\mathbf{P}\left\{\xi_{1}=k \mid \xi_{1} \leqslant r\right\}, \quad k=1,2, \ldots, r \\
& \mathbf{P}\left\{\tilde{\xi}_{1}^{(r)}=k\right\}=\mathbf{P}\left\{\xi_{1}=k \mid \xi_{1} \neq r\right\}, \quad k=1,2, \ldots
\end{aligned}
$$

Введем обозначения

$$
v_{N}^{(r)}=\xi_{1}^{(r)}+\ldots+\xi_{N}^{(r)}, \quad \tilde{v}_{N}^{r}=\tilde{\xi}_{1}^{(r)}+\ldots+\tilde{\xi}_{N}^{(r)}, \quad P_{r}=\mathbf{P}\left\{\xi_{1}>r\right\} .
$$

Из равенства (5) легко вывести известные (см. [6]) свойства обобщенной схемы размещения.

Лемма 1. Справедливы равенства

$$
\begin{aligned}
\mathbf{P}\left\{\eta_{(N)} \leqslant r\right\} & =\left(1-P_{r}\right)^{N} \frac{\mathbf{P}\left\{v_{N}^{(r)}=n\right\}}{\mathbf{P}\left\{v_{N}=n\right\}}, \\
\mathbf{P}\left\{\mu_{r}=k\right\} & =\left(\begin{array}{c}
N \\
k
\end{array}\right) p_{r}^{k}\left(1-p_{r}\right)^{N-k} \frac{\mathbf{P}\left\{\tilde{v}_{N-k}^{(r)}=n-k r\right\}}{\mathbf{P}\left\{v_{N}=n\right\}} .
\end{aligned}
$$

Лемма 1 будет использована для доказательства теорем 4-6. Так как асимптотика вероятности $\mathbf{P}\left\{v_{N}=n\right\}$ дана соотношениями (11)-(13), осталось рассмотреть предельное поведение бинома $\left(1-P_{r}\right)^{N}$, вероятностей $\mathbf{P}\left\{v_{N}^{(r)}=n\right\}$ и $\mathbf{P}\left\{\tilde{v}_{N-k}^{(r)}=n-k r\right\}$, и биномиальной вероятности $\left(\begin{array}{c}N \\ k\end{array}\right) p_{r}^{k}\left(1-p_{r}\right)^{N-k}$.

Лемма 2. Пусть $N \rightarrow \infty, r=(N / \gamma)^{1 / \tau}(1+o(1)), 0<\gamma<\infty$. Тогда $\left(1-P_{r}\right)^{N} \rightarrow e^{-\gamma}$.

Используя (2), нетрудно получить, что

$$
P_{r}=\mathbf{P}\left\{\xi_{1}>r\right\}=\sum_{k=r+1}^{\infty} p_{k}=(r+1)^{-\tau},
$$

поэтому из соотношения $r^{\tau}=(N / \gamma)(1+o(1))$ следует утверждение леммы. 
Поскольку $r \rightarrow \infty$ в теореме 4 , для доказательства локальной сходимости распределения суммы $v_{N}^{(r)}$ к предельным законам необходимо применить соответствующие теоремы для схемы серий. К сожалению, в нашем случае не выполняются известные простые достаточные условия такой сходимости (см., например, [11]) и мы будем использовать результаты работы [12], условия которых не всегда легко проверяемы.

Лемма 3. Пусть $N \rightarrow \infty, N P_{r} \rightarrow \gamma, 0<\gamma<\infty, \tau>2$. Тогда равномерно относительно цельх положительных $k$ таких, что $(k-N \zeta(\tau)) /(\sigma \sqrt{N})$ лежит в любом фиксированном конечном интервале,

$$
\mathbf{P}\left\{v_{N}^{(r)}=k\right\}=\frac{1+o(1)}{\sigma \sqrt{2 \pi N}} \exp \left\{-\frac{(k-N \zeta(\tau))^{2}}{2 \sigma^{2} N}\right\} .
$$

Доказательство. Обозначим $\varphi(t)$ характеристическую функцию распределения (2). Используя (4), нетрудно получить, что

$$
\varphi(t)=1+\left(e^{i t}-1\right) \Phi\left(e^{i t}, \tau, 1\right) .
$$

Обозначим характеристическую функцию случайной величины $\left(v_{N}^{(r)}-N \zeta(\tau)\right) /(\sigma \sqrt{N})$ через $\Psi_{1}(t)$. Тогда из (15) следует, что

$$
\Psi_{1}(t)=\exp \left\{-\frac{i t N \zeta(\tau)}{\sigma \sqrt{N}}\right\}\left(\frac{\varphi(t /(\sigma \sqrt{N}))-\sum_{k=r+1}^{\infty} p_{k} \exp \{i t k /(\sigma \sqrt{N})\}}{1-P_{r}}\right)^{N} .
$$

В силу (11) при любом фиксированном $t$

$$
\varphi^{N}\left(\frac{t}{\sigma \sqrt{N}}\right) \exp \left\{-\frac{i t N \zeta(\tau)}{\sigma \sqrt{N}}\right\} \rightarrow e^{-t^{2} / 2},
$$

поэтому

$$
\Psi_{1}(t)=e^{-t^{2} / 2}\left(\frac{1-\varphi^{-1}(t /(\sigma \sqrt{N})) \sum_{k=r+1}^{\infty} p_{k} \exp \{i t k /(\sigma \sqrt{N})\}}{1-P_{r}}\right)^{N}(1+o(1)) .
$$

Заметим, что при $k \rightarrow \infty$

$$
p_{k}=\tau / k^{\tau+1}(1+o(1)) \text {. }
$$

Учитывая (18) и соотношения

$$
N P_{r} \rightarrow \gamma, \quad \varphi(t /(\sigma \sqrt{N}))=1+o(1), \quad \exp \{i t k /(\sigma \sqrt{N})\}=1+\delta k / \sqrt{N}
$$

где величина $\delta$ не зависит от $k$ и может быть сделана сколь угодно малой при достаточно больших $N$, из (19) и (20) получаем, что

$$
\Psi_{1}(t) \rightarrow e^{-t^{2} / 2}
$$

Таким образом, распределение случайной величины $\left(v_{N}^{(r)}-N \zeta(\tau)\right) /(\sigma \sqrt{N})$ слабо сходится к стандартному нормальному закону. Докажем локальную сходимость.

Обозначим $\xi^{*}$ случайную величину, полученную из $\xi_{1}$ путем симметризации, то есть $\xi^{*}=\xi_{1}-\xi_{2}$. Пусть $d \in[1 / 4,1 / 2],\langle\alpha\rangle-$ расстояние от $\alpha$ до ближайшего целого числа. 
Нам потребуется величина $H_{N}=\inf _{d} H_{N}(d)$, где $H_{N}(d)=N \mathbf{E}\left\langle\xi^{*} d\right\rangle^{2}$. Используя (2), нетрудно найти, что при всех $\tau>0$ и $1 / 4 \leqslant d \leqslant 1 / 2$ справедливо неравенство

$$
H_{N} \geqslant C_{3} N \text {, }
$$

здесь и далее символы $C_{3}, C_{4}, \ldots$ означают некоторые положительные постоянные. Из этого неравенства и (10) следует, что

$$
\sigma^{2} N=O\left(H_{N}\right)
$$

Согласно первому условию теоремы 1 работы [12], соотношений (21) и (23) достаточно для того, чтобы утверждение леммы 3 было верным.

Лемма 4. Пусть $N \rightarrow \infty, N P_{r} \rightarrow \gamma, 0<\gamma<\infty, \tau=2$. Тогда равномерно относительно иельх положительных $k$ таких, что $(k-N \zeta(2)) / \sqrt{N \ln N}$ лежит в любом фиксированном конечном интервале,

$$
\mathbf{P}\left\{v_{N}^{(r)}=k\right\}=\frac{1+o(1)}{\sqrt{2 \pi N \ln N}} \exp \left\{-\frac{(k-N \zeta(2))^{2}}{2 N \ln N}\right\} .
$$

Доказательство. Из (12) ясно, что в условиях леммы

$$
\varphi^{N}\left(\frac{t}{\sqrt{N \ln N}}\right) \exp \left\{-\frac{i t N \zeta(2)}{\sqrt{N \ln N}}\right\} \rightarrow e^{-t^{2} / 2} .
$$

Отсюда получаем, повторяя рассуждения, приведенные при доказательстве леммы 3 , что

$$
\Psi_{2}(t) \rightarrow e^{-t^{2} / 2},
$$

где $\Psi_{2}(t)$ - характеристическая функция величины $\left(v_{N}^{(r)}-N \zeta(2)\right) / \sqrt{N \ln N}$. Введем обозначение

$$
B_{N}^{2}(u)=N \sum_{|k| \leqslant u} k^{2} \mathbf{P}\left\{\xi^{*}=k\right\}
$$

Согласно (2),

$$
\mathbf{P}\left\{\xi^{*}=k\right\}=p_{k+1} p_{1}+p_{k+2} p_{2}+\ldots
$$

Из (2), (25), (26) нетрудно получить, что

$$
B_{N}^{2}\left(H_{N}^{\beta}\right) \geqslant C_{4} N \ln N,
$$

где $\beta \in(0,1 / 2)$. Отсюда и из (22) следует, что

$$
H_{N} \rightarrow \infty, \quad N \ln N=O\left(B_{N}^{2}\left(H_{N}^{\beta}\right)\right) .
$$

Последние соотношения, вместе с (24), означают, что выполнено третье условие теоремы 1 из [12], откуда и следует лемма 4.

Лемма 5. Пусть $N \rightarrow \infty, N P_{r} \rightarrow \gamma, 0<\gamma<\infty, 1<\tau<2$. Тогда равномерно относительно цельх положительных $k$ таких, что $(k-N \zeta(\tau)) / N^{1 / \tau}$ лежит в любом фиксированном конечном интервале,

$$
\mathbf{P}\left\{v_{N}^{(r)}=k\right\}=\frac{1+o(1)}{N^{1 / \tau}} g\left(\frac{k-N \zeta(\tau)}{N^{1 / \tau}}\right),
$$

где $g(x)$ - плотность устойчивого распределения, определенного в (14). 
Доказательство. Из (13) и (14) следует, что

$$
\varphi^{N}\left(\frac{t}{N^{1 / \tau}}\right) \exp \left\{-\frac{i t N \zeta(\tau)}{N^{1 / \tau}}\right\} \rightarrow f(t)
$$

Отсюда находим, как и в лемме 3, что

$$
\Psi_{3}(t)=f(t)\left(\frac{1-\varphi^{-1}\left(t / N^{1 / \tau}\right) \sum_{k=r+1}^{\infty} p_{k} \exp \left\{i t k / N^{1 / \tau}\right\}}{1-P_{r}}\right)^{N}(1+o(1)) \rightarrow f(t),
$$

где $\Psi_{3}(t)$ - характеристическая функция случайной величины $\left(v_{N}^{(r)}-N \zeta(\tau)\right) / N^{1 / \tau}$. Достаточные условия локальной сходимости, приведенные в теореме 1 из [12], в нашем случае не выполнены, поэтому далее мы будем следовать обычной схеме доказательства локальных теорем. По формуле обращения

$$
\mathbf{P}\left\{v_{N}^{(r)}=k\right\}=\frac{1}{2 \pi N^{1 / \tau}} \int_{-\pi N^{1 / \tau}}^{\pi N^{1 / \tau}} \exp \left\{-\frac{i t k}{N^{1 / \tau}}\right\} \Psi_{3}(t) d t
$$

Поскольку

$$
g(x)=\frac{1}{2 \pi} \int_{-\infty}^{\infty} e^{-i t x} f(t) d t
$$

получаем, что

$$
2 \pi N^{1 / \tau} \mathbf{P}\left\{v_{N}^{(r)}=k\right\}-2 \pi g\left((k-N \zeta(\tau)) / N^{1 / \tau}\right)=I_{1}+I_{2}+I_{3}+I_{4},
$$

где

$$
\begin{aligned}
& I_{1}=\int_{-A}^{A} \exp \left\{-\frac{i t k}{N^{1 / \tau}}\right\}\left(\Psi_{3}(t)-f(t)\right) d t, \quad I_{2}=\int_{A<|t| \leqslant \varepsilon N^{1 / \tau}} \exp \left\{-\frac{i t k}{\left.N^{1 / \tau}\right\} \Psi_{3}(t) d t,}\right. \\
& I_{3}=\int_{\varepsilon N^{1 / \tau}<|t| \leqslant \pi N^{1 / \tau}} \exp \left\{-\frac{i t k}{N^{1 / \tau}}\right\} \Psi_{3}(t) d t, \quad I_{4}=-\int_{A<|t|} f(t) d t,
\end{aligned}
$$

выбор положительных постоянных $A$ и $\varepsilon$ будет ясен из дальнейшего. Лемма будет доказана, если мы покажем, что каждый из интегралов $I_{1}, \ldots, I_{4}$ стремится к нулю.

Ясно, что $I_{1} \rightarrow 0$ в силу (28). Из (14) нетрудно получить, что

$$
\left|I_{4}\right| \leqslant \int_{A}^{\infty} e^{-C_{5}|t|^{\tau}} d t
$$

и последнее выражение может быть сделано сколь угодно малым выбором достаточно большого $A$. Из (27), (28) следует, что в области интегрирования $I_{2}$

$$
\left|\Psi_{3}(t)\right| \leqslant C_{6} \varphi^{N}\left(t / N^{1 / \tau}\right) .
$$

Используя известную [10] асимптотику функции (4) при $z \rightarrow 0$

$$
\Phi\left(e^{z}, \tau, 1\right)=\zeta(\tau)+\Gamma(1-\tau)(-z)^{\tau-1}+O(z),
$$

из (4), (18) и (29) находим, что

$$
\left|\Psi_{3}(t)\right| \leqslant C_{6} \exp \left\{-C_{7}|t|^{\tau-1} N^{1 / \tau}\right\} .
$$


Из последнего неравенства следует, что $I_{2} \rightarrow 0$. Нам осталось рассмотреть $I_{3}$. Ясно, что

$$
\Psi_{3}(t)=\left(\frac{\varphi\left(t / N^{1 / \tau}\right)-\sum_{k=r+1}^{\infty} p_{k} \exp \left\{i t k / N^{1 / \tau}\right\}}{1-P_{r}}\right)^{N} .
$$

В области интегрирования интеграла $I_{3}$, как известно, справедливо неравенство

$$
\left|\varphi\left(t / N^{1 / \tau}\right)\right| \leqslant e^{-C_{8}},
$$

поэтому из (30) следует, что

$$
\left|I_{3}\right| \leqslant 2 \pi N^{1 / \tau}\left(\frac{e^{-C_{8}}+P_{r}}{1-P_{r}}\right)^{N} \rightarrow 0 .
$$

Лемма 5 доказана.

Далее мы рассмотрим предельное поведение суммы $\tilde{v}_{N}^{(r)}$. Из (2), (10) и (16) следует, что

$$
\begin{aligned}
m_{r} & =\mathbf{E} \tilde{\xi}_{1}^{(r)}=\left(m-r p_{r}\right) /\left(1-p_{r}\right), \\
\sigma_{r}^{2} & =\mathbf{D} \tilde{\xi}_{1}^{(r)}=\frac{\sigma^{2}}{\left(1-p_{r}\right)^{2}}\left(1-p_{r}-\frac{(m-r)^{2}}{\sigma^{2}} p_{r}\right) .
\end{aligned}
$$

Нетрудно видеть, что при фиксированных $r$ имеют место соотношения, аналогичные (11)-(13), откуда получаем следующее утверждение.

Лемма 6. При $N \rightarrow \infty$ справедливы следующие утверждения.

1. Если $\tau>2$, то

$$
\sup _{k}\left|\sigma_{r} \sqrt{2 \pi N} \mathbf{P}\left\{\tilde{v}_{N}^{(r)}=k\right\}-\exp \left\{-\frac{\left(k-N m_{r}\right)^{2}}{2 \sigma_{r}^{2} N}\right\}\right| \rightarrow 0 .
$$

2. Если $\tau=2$, то

$$
\sup _{k}\left|\sqrt{2 \pi N \ln N} \mathbf{P}\left\{\tilde{v}_{N}^{(r)}=k\right\}-\exp \left\{-\frac{\left(k-N m_{r}\right)^{2}}{2 N \ln N}\right\}\right| \rightarrow 0 .
$$

3. Если $1<\tau<2$, то

$$
\sup _{k}\left|N^{1 / \tau} \mathbf{P}\left\{\tilde{v}_{N}^{(r)}=k\right\}-g\left(\frac{k-N m_{r}}{N^{1 / \tau}}\right)\right| \rightarrow 0,
$$

где $g(x)$ - плотность устойчивого распределения, определенного в (13).

Теперь мы можем доказать теоремы 4-6. Если выполнено первое условие теоремы 4, то из леммы 3 и соотношения (11) находим, что

$$
\mathbf{P}\left\{v_{N}^{(r)}=n\right\} / \mathbf{P}\left\{v_{N}=n\right\} \rightarrow 1 .
$$

Из лемм 4, 5 и, соответственно, соотношений (12), (13), находим, что (33) остается в силе и в условиях 2, 3 теоремы 4. Тогда эта теорема очевидным образом следует из первого утверждения леммы 1 , леммы 2 и (33). 
Пусть выполнены условия теоремы 5. Согласно известному нормальному приближению для биномиального распределения при $N p_{r}\left(1-p_{r}\right) \rightarrow \infty$

$$
\left(\begin{array}{l}
N \\
k
\end{array}\right) p_{r}^{k}\left(1-p_{r}\right)^{N-k}=\frac{1+o(1)}{\sqrt{2 \pi N p_{r}\left(1-p_{r}\right)}} \exp \left\{-\frac{\left(k-N p_{r}\right)^{2}}{2 N p_{r}\left(1-p_{r}\right)}\right\}
$$

равномерно относительно $\left(k-N p_{r}\right) / \sqrt{N p_{r}\left(1-p_{r}\right)}$ в любом фиксированном конечном интервале. Утверждение теоремы 5 нетрудно получить прямыми вычислениями с помощью (11), (31), (32), (34), второго утверждения леммы 1 и леммы 6. При выполнении первого условия теоремы 6 вероятность $\mathbf{P}\left\{v_{N}=n\right\}$ можно оценить с помощью (12). Это же соотношение используем и для оценки $\mathbf{P}\left\{\tilde{v}_{N-k}^{(r)}=n-k r\right\}$, учитывая (31) и то, что нормирующий множитель предельного распределения, согласно теореме 2.6.2 в [7], имеет вид $((N-k) \ln (N-k)) /\left(1-p_{r}\right)$. Отсюда следует, что

$$
\mathbf{P}\left\{\tilde{v}_{N-k}^{(r)}=n-k r\right\} / \mathbf{P}\left\{v_{N}=n\right\} \rightarrow 1
$$

и из (34) и леммы 1 получаем утверждение теоремы 6. При выполнении второго условия теорема 6 доказывается аналогично с использованием соотношений (13), (31), (34), (35), леммы 1 и теорем 2.1.1, 2.6.1 из [7].

\section{Список литературы}

1. Cheplyukova I., Pavlov Yu., Limit distributions of vertex degree in conditional power-law random graphs. In: Trans. XXVI International Seminar on Stability Problems for Stochastic Models. Ort Braude College, Karmel, Israel, 2007, pp. 52-59.

2. Павлов Ю. Л., Чеплюкова И. А., Случайные графы Интернет-типа и обобщенная схема размещения. Дискретная математика (2008) 20, №3, 3-18.

3. Reittu H., Norros I., On the power-law random graph model of massive data networks. Performance Evaluation (2004) 55, 3-23.

4. Faloutsos C., Faloutsos P., Faloutsos M., On power-law relationships of the internet topology. Computer Communications Rev. (1999) 29, 251-252.

5. Newman M. E. J., Strogatz S. H., Watts D. J., Random graphs with arbitrary degree distribution and their applications. Phys. Rev. E (2001) 64, 026118.

6. Колчин В. Ф., Случайные графы. Физматлит, Москва, 2000.

7. Ибрагимов И. А., Линник Ю. В., Независимые и стационарно связанные величины. Наука, Москва, 1965.

8. Павлов Ю. Л., Предельное распределение объема гигантской компоненты в случайном графе Интернет-типа. Дискретная математика (2007) 19, №3, 22-34.

9. Robinson J. E., Note on the Bose-Einstein integral functions. Phys. Rev. 2 (1951) 83, 678-679.

10. Wood D., Techn. Rep. 15-20, Univ. Kent, 1992.

11. Колчин А. В., Предельные теоремы для обобщенной схемы размещения. Дискретная математика (2003) 15, №4, 148-157.

12. Мухин А. Б., Локальные предельные теоремы для решетчатых случайных величин. Теория вероятностей и ее применения (1991) 36, №4, 660-674.

13. Боровков А. А., Курс теории вероятностей. Наука, Москва, 1972.

Статья поступила 24.03.2008. 